\title{
Correction to: 3D Parallel Multigrid Methods for Real-Time Fluid Simulation
}

\author{
Feifei Wan · Yong Yin · Suiyun Zhang
}

Published online: 6 April 2018

(c) 3D Research Center, Kwangwoon University and Springer-Verlag GmbH Germany, part of Springer Nature 2018

Correction to: 3D Res (2018) 9:8

https://doi.org/10.1007/s13319-018-0157-z

Unfortunately, the co-author's given name has been published incorrectly in the original online publication. The correct given name should be: Suiyun.

The original article can be found online at https://doi.org/10.1007/s13319-018-0157-z.

F. Wan · Y. Yin $(\bowtie)$

Marine Dynamic Simulation and Control Laboratory of Dalian Maritime University, Dalian, China

e-mail: wff@dlmu.edu.cn

Y. Yin

e-mail: bushyin@163.com

S. Zhang

School of Software, Tsinghua University, Beijing, China

e-mail: zhangsuiyun13@mails.tsinghua.edu.cn 